\title{
Models to predict the short-term survival of acute-on-chronic liver failure patients following liver transplantation
}

\author{
Min Yang, Bo Peng, Quan Zhuang, Junhui Li, Hong Liu, Ke Cheng and Yingzi Ming ${ }^{*}$
}

\begin{abstract}
Background: Acute-on-chronic liver failure (ACLF) is featured with rapid deterioration of chronic liver disease and poor short-term prognosis. Liver transplantation (LT) is recognized as the curative option for ACLF. However, there is no standard in the prediction of the short-term survival among ACLF patients following LT.

Method: Preoperative data of 132 ACLF patients receiving LT at our center were investigated retrospectively. Cox regression was performed to determine the risk factors for short-term survival among ACLF patients following LT. Five conventional score systems (the MELD score, ABIC, CLIF-C OFs, CLIF-SOFAs and CLIF-C ACLFs) in forecasting shortterm survival were estimated through the receiver operating characteristic (ROC). Four machine-learning (ML) models, including support vector machine (SVM), logistic regression (LR), multi-layer perceptron (MLP) and random forest (RF), were also established for short-term survival prediction.
\end{abstract}

Results: Cox regression analysis demonstrated that creatinine (Cr) and international normalized ratio (INR) were the two independent predictors for short-term survival among ACLF patients following LT. The ROC curves showed that the area under the curve (AUC) ML models was much larger than that of conventional models in predicting shortterm survival. Among conventional models the model for end stage liver disease (MELD) score had the highest AUC (0.704), while among ML models the RF model yielded the largest AUC (0.940).

Conclusion: Compared with the traditional methods, the ML models showed good performance in the prediction of short-term prognosis among ACLF patients following LT and the RF model perform the best. It is promising to optimize organ allocation and promote transplant survival based on the prediction of ML models.

Keywords: ACLF, Machine-learning models, Liver transplantation, Prognosis, MELD

\section{Background}

Acute-on-chronic liver failure (ACLF) is a syndrome with acute exacerbation of chronic hepatopathy, characterized by intense systemic inflammation, multiple organ dysfunction, and poor prognosis [1-3]. Liver transplantation (LT) is regarded as the curative method for terminal liver diseases including ACLF $[4,5]$. However,

*Correspondence: mingyz_china@csu.edu.cn

Transplantation Center, Third Xiangya Hospital of Central South University, Changsha, Hunan, People's Republic of China there is considerable discrepancy between the increasing ACLF patients waiting for LT and the shortage of available organ donors, and the 1-year post-transplantation mortality rate of ACLF still reaches approximately $20 \%$ [6]. Consequently it is necessary to establish the selection criteria of ACLF for LT, which may improve organ allocation and transplant outcome.

In previous studies, several scoring systems were applied to forecast the short-term outcome among ACLF patients. The model for end stage liver disease (MELD) score accurately evaluates the liver conditions and 
prognosis of terminal stage hepatopath, including ACLF, which had important implications for organ-allocation in emergency [7]. However, some studies have indicated a weak association between pre-transplant MELD score and post-transplant survival $[8,9]$. The predictive value of other scores directed at ACLF, including the Chronic Liver Failure Consortium Organ Failure scores (CLIF-C OFs), CLIF sequential organ failure assessment scores (CLIF-SOFAs) and CLIF Consortium ACLF scores (CLIF-C ACLFs), has also been validated in ACLF patients [10-12]. However, few studies revealed these scores have good predictive value for short-term outcome in ACLF patients following LT. Therefore, it is essential to generate a new accurate prediction model for postoperative survival of ACLF following LT.

Machine learning (ML) leverages software algorithms to identify patterns in large data sets to establish predictive models more precisely than conventional methods. Machine learning algorithms can find novel patterns between variables and generate predictions by learning from multiple features simultaneously [13]. Several recent studies have indicated that ML models are useful for improving organ allocation and the transplant outcome after LT [14-18]. Previously our team had applied eight ML models for tacrolimus dose requirement post kidney transplantation [19]. The application of ML models is promising to forecast the short-term transplant survival of ACLF patients, which may contribute to organ allocation and benefit prognosis.

In the study, my team retrospectively analyzed ACLF patients receiving LT in our institution, and comparing the predictive value of conventional models and ML models for predicting 90-day posttransplant survival of these patients based on preoperative variables.

\section{Methods}

\section{Study design}

A retrospective study was designed to develop ML models to make a prediction for short-term prognosis in ACLF patients following LT. ACLF patients undergoing LT were enrolled from the Transplantation Center, Third Xiangya Hospital, Central South University between March, 2012 and December, 2019. The study protocol complied with the standards of the Declaration of Helsinki and obtained approval from the Institutional Review Board of Third Xiangya Hospital, Central South University (No. 2020-S398).

\section{Patients}

Liver grafts with $<30 \%$ macrovesicular steatosis from donation after cardiac death (DCD) were all approved and distributed by China Organ Transplant Response System (COTRS). All recipients were administered
Basiliximab as intraoperative induction therapy. The standard maintenance immunosuppressions consisted of calcineurin inhibitors (CNIs; tacrolimus or cyclosporin), mycophenolate mofetil or mycophenolate sodium (MMF), and prednisone. According to the criteria of the Asian Pacific Association for the Study of the Liver (APASL), ACLF was defined as "acute hepatic insult manifesting as jaundice (serum bilirubin $>5 \mathrm{mg} /$ $\mathrm{dL}$ ) and coagulopathy (INR $>1.5$ ), complicated within 4 weeks by ascites and/or encephalopathy in a patient with previously diagnosed or undiagnosed chronic liver disease" [20]. Patient short-term survival was defined as 90 days' postoperative survival of ACLF patients. Preoperative variables including clinical characteristics and biochemical parameters were collected and analyzed. For patients with multiple biological data, the worst value during hospitalization in our department before LT was selected. All ACLF patients' preoperative clinical data were used to calculate five conventional prediction formulas (the MELD score, ABIC, CLIF-C OFs, CLIFSOFAs and CLIF-C ACLFs). The details of the formulas for the scores are shown in Additional file 1: Supplementary Table 1. All patients provided informed consent to participate in the study.

\section{Model building}

Four ML classifiers were employed to predict 90-day post-transplant survival based on the patient's preoperative variables: Support vector machine (SVM), logistic regression (LR), multilayer perceptron (MLP) and random forest (RF). The ML models were trained to build a prediction model using fivefold cross-validation and implemented via Python programming language (version 3.6) and Scikit-learn package (version 0.22 ) as previously reported [21]. In order to estimate the performance of different ML models, we applied $\mathrm{k}$-fold cross-validation (with $\mathrm{k}=5$ ) and selected the good hyperparameters. In general, 132 patients were divided at random into five subgroups. A single subgroup is retained as the validation cohort for testing the final selected model while 4 subgroups are used as derivation cohort to create a formula. The average area under the curve (AUC) was computed by five independent runs. Finally, the algorithms were developed in the full data set of the eligible group.

According to the SVM model, the function was $\mathrm{f}(\mathrm{x})=\operatorname{SIGN}\left(\beta_{0}+\beta_{\mathrm{m}} \mathrm{x}_{\mathrm{m}}\right)$. As the value was 1 , the patient was divided into the death group. As the value was -1 , the patient was divided into the survival group. According to the LR model, the function was $\mathrm{f}(\mathrm{x})=$ SIGMOID $\left(\beta_{0}+\beta_{\mathrm{m}} \mathrm{x}_{\mathrm{m}}\right)$. As the value was over 0.5 , the patient was divided into the death group. As the value was no more than 0.5 , the patient was divided into the survival group. The RF model was built with ten trees. Each tree in the 
forest is trained using a diverse portion of the database. Majority voting was performed to obtain the final predictions.

\section{Statistical analysis}

Descriptive statistics are represented using means \pm standard deviations (SD) or interquartile range (IQR) for continuous data and percentages for count data. Preoperative data were compared by Student's t-test, the Pearson Chisquared or Fisher exact test as appropriate. Kaplan-Meier (KM) survival curves and Cox regression analysis were made to analyze the risk factors for 90-day prognosis among ACLF patients following LT. The predictive power of conventional score systems for 90-day outcome was estimated by the receiver operating characteristic (ROC) curve. Results of $P<0.05$ were thought to be significant and statistical calculations were performed using in SPSS version 22.0 (SPSS, Inc., Chicago, IL, USA) and GraphPad Prism 5 software (GraphPad Software, Inc., San Diego, CA).

\section{Results}

\section{Basic characteristics}

A total of 271 patients accepted LT at our institution between March 1st, 2012 and December 31st, 2019, of which 132 patients achieving the APASL criteria of ACLF were eligible for this study. The flowchart for inclusion is shown in Fig. 1. All the patients included in the study also met the European Association for the Study of Liver (EASL)-Chronic Liver Failure (EASL-CLIF) Consortium ACLF criteria. The EASL-CLIF ACLF grades were shown in Table 1 and the detailed information was shown in Additional file 1: Supplementary Table 2. Nineteen ACLF patients (14.4\%) died within 90 days after liver transplantation. The causes of death were upper gastrointestinal bleeding $(n=7,36.8 \%)$, multiple organ dysfunction syndrome (MODS) $(n=6,31.5 \%)$, severe pneumonia $(\mathrm{n}=3,15.8 \%)$, abdominal infection $(\mathrm{n}=1,5.3 \%)$, heart arrest $(n=1,5.3 \%)$ and cerebral herniation $(n=1,5.3 \%)$.

Comparison of clinical data between the survival group and the death group displayed no statistical difference in terms of age, sex, etiology, EASL-CLIF ACLF grade, total bilirubin (TBiL), creatinine $(\mathrm{Cr})$, international normalized ratio (INR), hepatic encephalopathy (HE), prothrombin time (PT), urea, albumin, direct bilirubin (DBIL), hemoglobin, platelet count, white blood cells (WBC), neutrophil count (NEUT) and lymphocyte count (LYM) (Table 1). The characteristics of donors for the two groups also showed no significant difference, which was shown in the Additional file 1: Supplementary Table 3.

\section{Patient survival analysis}

Based on traditional formulae scores, the cut-off values of the important score-related parameters $(\mathrm{Cr}$,
INR, TBiL, Plt and WBC) were determined, which were applied for Kaplan-Meier (KM) survival analysis. The results indicated that the post-transplant mortality among ACLF patients was significantly associated with higher values of $\mathrm{Cr}(\mathrm{Cr} \geq 132.6 \mu \mathrm{mol} / \mathrm{L})$ and INR $(\mathrm{INR} \geq 2.0)(P<0.05)$. The differences among other factors were not statistically significant (Fig. 2).

The model of Cox regression was applied to identify the independent risk factors for short-term outcome. Univariate Cox regression analysis revealed that $\operatorname{Cr}(P=0.001)$ and $\operatorname{INR}(P=0.034)$ were poor prognostic indicators for ACLF patients following LT. Factors with $P<0.15$ were further analyzed in multivariate cox regression. The results of multivariate analysis displayed that $\mathrm{Cr}$ (HR, 1.006; 95\% CI, 1.001-1.011; $P=0.030)$ and INR (HR, 1.454; 95\% CI, 1.100-1.921; $P=0.009)$ were independent prognostic markers of short-term outcome (Table 2).

\section{Predictive value of conventional models}

In comparison to those in the survival group, the scores of conventional models, including the MELD score, ABIC, CLIF-C OFs, CLIF-SOFAs, and CLIF-C ACLFs were higher in the death group. However, only MELD score $(P=0.01)$ and CLIF-C ACLFs $(P=0.04)$ showed significance between the survival group and death group (Fig. 3 and Table 3). According to the ROC analysis, the area under a receiver operating characteristics (AUROC) of MELDs (AUROC: 0.704) was higher than those of ABIC (AUROC: 0.607), CLIF-C OFs (AUROC: 0.606), CLIF-C ACLFs (AUROC: 0.653), and CLIF-SOFAs (AUROC: 0.633) for prediction of the 90-day outcome in ACLF patients following LT.

\section{Predictive value of ML models}

Four ML models (SVM, LR, MLP and RF) were trained and compared to improve the prediction performance. All ML models had good performance in terms of AUROC (Fig. 4), higher than those of conventional models. Among the ML models, the RF model had the highest AUROC of 0.94. The final result of RF model was derived from majority voting by the ten trees. The AUROCs of SVM, LR and MLP were 0.81, 0.83 and 0.89, respectively (Fig. 5). Cr, INR, etiology, DBiL, LYM and NEUT were chosen to develop the SVM and LR models. The coefficients of parameters in the models are described in Table 4. In the two models, the coefficients of $\mathrm{Cr}$ and INR are negative, indicating a negative correlation with the prognosis in ACLF patients following LT. The other parameters including etiology of liver disease, DBIL, LYM and NEUT, are positively associated with transplant outcome. 
Table 1 Clinical characteristics of the patients

\begin{tabular}{|c|c|c|c|c|}
\hline Characteristics & $\operatorname{ALL}(n=132)$ & Survival( $n=113)$ & $\operatorname{Death}(n=19)$ & $P$ value \\
\hline Age, mean $\pm S D$ (years) & $45.92 \pm 11.07$ & $45.50 \pm 11.04$ & $48.37 \pm 11.26$ & 0.30 \\
\hline Etiologies, $n(\%)$ & & & & 0.29 \\
\hline HBV & $111(84.1 \%)$ & $96(85.0 \%)$ & $15(78.9 \%)$ & \\
\hline Cholestasis & $6(4.5 \%)$ & $4(3.5 \%)$ & $2(10.5 \%)$ & \\
\hline Alcohol & $7(5.3 \%)$ & $7(6.2 \%)$ & $0(0 \%)$ & \\
\hline Other & $8(6.1 \%)$ & $2(1.8 \%)$ & $6(31.6 \%)$ & \\
\hline Male, n(\%) & $108(81.8 \%)$ & $94(83.2 \%)$ & $14(73.7 \%)$ & 0.51 \\
\hline \multicolumn{5}{|l|}{ ACLF Score } \\
\hline CLIF-ACLF grade, $n(\%)$ & & & & 0.22 \\
\hline Grade 1 & $5(3.8 \%)$ & $5(4.4 \%)$ & $0(0 \%)$ & \\
\hline Grade 2 & $91(68.9 \%)$ & $80(70.8 \%)$ & $11(57.9 \%)$ & \\
\hline Grade 3 & $36(27.3 \%)$ & $28(24.8 \%)$ & $8(42.1 \%)$ & \\
\hline TBILI, mean \pm SD $(\mu \mathrm{mol} / \mathrm{L})$ & $401.64 \pm 198.29$ & $404.00 \pm 201.33$ & $387.61 \pm 183.55$ & 0.74 \\
\hline $\mathrm{Cr}$, mean $\pm \mathrm{SD}(\mu \mathrm{mol} / \mathrm{L})$ & $84.71 \pm 51.07$ & $78.23 \pm 37.09$ & $123.26 \pm 92.67$ & 0.05 \\
\hline $\mathrm{INR}$, mean $\pm \mathrm{SD}$ & $2.53 \pm 1.22$ & $2.44 \pm 1.13$ & $3.07 \pm 1.58$ & 0.11 \\
\hline $\mathrm{HE}, \mathrm{n}(\%)$ & $29(22.0 \%)$ & $23(20.4 \%)$ & $6(31.6 \%)$ & 0.27 \\
\hline $\mathrm{PT}$, mean $\pm \mathrm{SD}(\mathrm{s})$ & $28.98 \pm 13.95$ & $28.28 \pm 13.28$ & $33.19 \pm 17.24$ & 0.25 \\
\hline Urea, mean $\pm \mathrm{SD}(\mathrm{mmol} / \mathrm{L})$ & $7.83 \pm 11.60$ & $7.04 \pm 11.20$ & $12.54 \pm 13.11$ & 0.06 \\
\hline Alb, mean $\pm S D(g / L)$ & $34.56 \pm 5.68$ & $34.43 \pm 5.76$ & $35.34 \pm 5.30$ & 0.52 \\
\hline $\mathrm{DBiL}, \operatorname{mean} \pm \mathrm{SD}(\mu \mathrm{mol} / \mathrm{L})$ & $239.56 \pm 131.65$ & $244.93 \pm 133.54$ & $207.62 \pm 117.93$ & 0.26 \\
\hline $\mathrm{Hb}$, mean $\pm \mathrm{SD}(\mathrm{g} / \mathrm{L})$ & $105.07 \pm 23.52$ & $105.44 \pm 23.19$ & $102.90 \pm 25.98$ & 0.67 \\
\hline Plt, mean $\pm S D(10 \wedge 3 c e l l s / \mu \mathrm{L})$ & $92.53 \pm 78.81$ & $93.78 \pm 82.80$ & $85.05 \pm 49.73$ & 0.66 \\
\hline WBC, mean $\pm \mathrm{SD}(10 \wedge 3$ cells $/ \mu \mathrm{L})$ & $8.06 \pm 4.42$ & $7.80 \pm 4.19$ & $9.59 \pm 5.49$ & 0.10 \\
\hline LYM, mean \pm SD $(10 \wedge 3 c e l l s / \mu \mathrm{L})$ & $1.09 \pm 0.77$ & $1.12 \pm 0.78$ & $0.92 \pm 0.70$ & 0.31 \\
\hline NEUT, mean \pm SD $(10 \wedge 3$ cells $/ \mu \mathrm{L})$ & $6.21 \pm 3.80$ & $5.95 \pm 3.71$ & $7.78 \pm 4.05$ & 0.05 \\
\hline Intensive Care Unit, n (\%) & $25(18.9 \%)$ & $18(15.9 \%)$ & $7(36.8 \%)$ & 0.031 \\
\hline Renal replacement therapy, n (\%) & $7(5.3 \%)$ & $3(2.7 \%)$ & $4(21.1 \%)$ & 0.001 \\
\hline Multidrug resistant organism infection, n (\%) & $3(2.3 \%)$ & $2(1.8 \%)$ & $1(5.3 \%)$ & 0.344 \\
\hline Donor age, years & $39.78 \pm 13.25$ & $40.50 \pm 12.90$ & $35.42 \pm 14.82$ & 0.51 \\
\hline Cause of death & & & & 0.09 \\
\hline Trauma & $68(51.5 \%)$ & $60(53.1 \%)$ & $8(42.1 \%)$ & \\
\hline Cerebrovascular accident (CVA) & $49(37.1 \%)$ & $43(38.1 \%)$ & $6(31.6 \%)$ & \\
\hline Other & $15(11.4 \%)$ & $10(8.8 \%)$ & $5(26.3 \%)$ & \\
\hline Donor risk index & $1.40 \pm 0.27$ & $1.40 \pm 0.23$ & $1.38 \pm 0.22$ & 0.37 \\
\hline Steatosis & & & & 0.38 \\
\hline$<5 \%$ & $38(28.8 \%)$ & $30(26.5 \%)$ & $8(42.1 \%)$ & \\
\hline $5 \% \sim 15 \%$ & $70(53.0 \%)$ & $62(54.9 \%)$ & $8(42.1 \%)$ & \\
\hline $15 \% \sim 30 \%$ & $24(18.2 \%)$ & $21(18.6 \%)$ & $3(15.8 \%)$ & \\
\hline Cold ischemia time & $7.52 \pm 2.01$ & $7.45 \pm 1.97$ & $7.89 \pm 2.28$ & 0.64 \\
\hline
\end{tabular}

Tested by Student's t-test or Pearson's chi-squared (X2) test; SD Standard Deviation; HBV Hepatitis B Virus; TBiL total bilirubin; Cr creatinine; INR International Normalized Ratio; HE hepatic encephalopathy; PT prothrombin time; Alb albumin; DBiL Direct Bilirubin; Hb hemoglobin; Plt platelet; WBC White Blood Cells; LYM lymphocyte count; NEUT neutrophil count

\section{Discussion}

This study successfully established four ML models for forecasting the short-term survival of ACLF patients following LT. The ML model had better performance than the conventional models, and the RF model best predicted the short-term survival of ACLF patients following
LT. ML algorithms could be a useful tool, facilitating better organ allocation and transplant outcomes.

Reportedly, there are several conventional models available to accurately estimate liver function and prognosis of patients with liver disease, such as Child-Pugh scores and the MELD. The allocation of donor Liver is 
271 patients with liver disease underwent liver transplantation from 2012.3 to 2019.12

Diagnostic criteria of acute-on-chronic liver failure (ACLF):

1. Acute decompensation (AD) of chronic liver diseases;

2. Jaundice (serum bilirubin $>5 \mathrm{mg} / \mathrm{dL}$ )

3. Coagulopathy (international normalized ratio $>1.5$ )

4. Within four weeks by ascites or encephalopathy

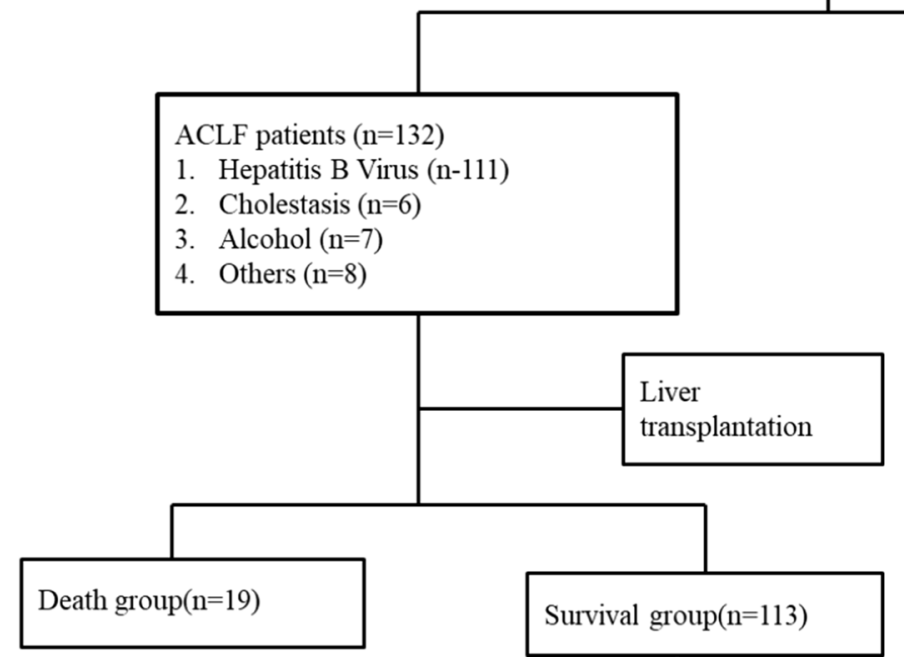

Patients not meet diagnostic criteria $(\mathrm{n}=139)$

1. Chronic B-related Hepatitis $(\mathrm{n}=42)$

2. Alcoholic hepatitis( $(n=28)$

3. Hepatocellular carcinoma $(\mathrm{n}=30)$

4. Others $(n=39)$

Fig. 1 Flowchart of the search strategy and selection of studies for inclusion

based on the recipient's MELD score, which has created a shift in donor liver allocation to the sickest recipients in order to minimize waiting list mortality [7, 22]. The MELD score is is widely used as a scoring system for organ allocation in liver transplantation and is the current standard prognostic tool for assessing 3- to 6-month survival in patients with hepatic failure [22]. However, ACLF has a unique clinical feature, and the predictor of MELD score for ACLF patients is not ideal. Liang Chen et al. exhibited that the ABIC score performed better than the MELD score in forecasting short-term survival among HBV-related ACLF patients [23]. Novel predictive assessment models (CLIF-C OFs, CLIF-SOFAs and CLIF-C ACLFs) have been developed and validated to forecast mortality in patients with ACLF, among which the CLIF-C ACLFs achieved better predictive accuracy than the MELD score [6].

Many previous researchers concluded that the MELD score and its exceptions had a limited ability to predict post-transplant mortality [24]. The MELD score could be correlated with post-transplant survival, but the pre-transplant MELD score appeared to have limited predictive ability [8]. No further studies showed that CLIF-C OFs, CLIF-SOFAs and CLIF-C ACLFs had good predictive value for short-term survival after LT. Our study indicated that the MELD score had a better AUROC (0.704) than other conventional models, but they all showed poor discrimination power in predicting postoperative survival.

Compared with traditional methods, ML algorithms utilize artificial intelligence to generate predictive models more precisely through the simultaneous detection of multidimensional parameters simultaneously [13]. The training set is employed to perform feature selection and parametric estimation, and the validation set is applied to assess the predictive power of the models. Furthermore, the model has the ability to self-evolve to adjust its structure when any errors are encountered. The models are promising in big data analysis, with the improvement of models performance using more data. Lau L et al. reported that RF model based on 15 donor and recipient variables had an excellent AUROC of 0.818 in forecasting 
Table 2 Univariate and multivariate Cox regression analyses of 90-day mortality

\begin{tabular}{|c|c|c|c|c|}
\hline \multirow[t]{2}{*}{ Characteristics } & \multicolumn{2}{|c|}{ Univariate } & \multicolumn{2}{|c|}{ Multivariate } \\
\hline & $P$ value & HR $(95 \% \mathrm{Cl})$ & $P$ value & HR $(95 \% \mathrm{Cl})$ \\
\hline Age, years & 0.308 & $1.023(0.979 \sim 1.068)$ & & \\
\hline HBV & 0.481 & $0.673(0.223 \sim 2.027)$ & & \\
\hline Male & 0.354 & $0.617(0.222 \sim 1.714)$ & & \\
\hline TBILI, $\mu \mathrm{mol} / \mathrm{L}$ & 0.740 & $1.000(0.997 \sim 1.002)$ & & \\
\hline $\mathrm{Cr}, \mu \mathrm{mol} / \mathrm{L}$ & 0.001 & $1.007(1.003 \sim 1.011)$ & 0.030 & $1.006(1.001 \sim 1.011)$ \\
\hline INR & 0.034 & $1.347(1.023 \sim 1.773)$ & 0.009 & $1.454(1.100 \sim 1.921)$ \\
\hline HE & 0.258 & $1.747(0.664 \sim 4.598)$ & & \\
\hline PT, s & 0.152 & $1.019(0.993 \sim 1.045)$ & & \\
\hline Urea, mmol/L & 0.071 & $1.019(0.998 \sim 1.040)$ & 0.257 & $1.019(0.986 \sim 1.054)$ \\
\hline Alb, g/L & 0.507 & $1.026(0.950 \sim 1.109)$ & & \\
\hline DBiL, $\mu \mathrm{mol} / \mathrm{L}$ & 0.265 & $0.998(0.994 \sim 1.002)$ & & \\
\hline $\mathrm{Hb}, \mathrm{g} / \mathrm{L}$ & 0.670 & $0.996(0.977 \sim 1.015)$ & & \\
\hline Plt, 10^3cells/ $/ \mathrm{L}$ & 0.664 & $0.999(0.992 \sim 1.005)$ & & \\
\hline 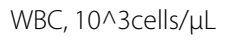 & 0.113 & $1.068(0.984 \sim 1.158)$ & 0.284 & $0.743(0.431 \sim 1.279)$ \\
\hline 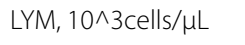 & 0.317 & $0.692(0.336 \sim 1.424)$ & & \\
\hline NEUT, 10^3cells/ $\mu \mathrm{L}$ & 0.060 & $1.095(0.996 \sim 1.203)$ & 0.150 & $1.582(0.847 \sim 2.955)$ \\
\hline
\end{tabular}

the risk of liver graft failure in LT, thereby providing further evidence that the application of ML tools contributes to improving organ allocation decisions and transplant outcomes [14]. Hyung-Chul Lee et al. applied seven ML methods to predict acute kidney injury (AKI) after LT and the gradient boosting machine model exhibited the best performance with the AUROC of 0.90 [16]. Our team developed ML methods to confirm the relation between peripheral lymphocyte subsets and pneumonia among kidney transplant recipients for better individualized therapy [21]. Therefore, the ML technique could be a powerful and promising means in the evaluating of the prognosis of ACLF following LT. Our results showed that ML models had a better performance than conventional models, and RF model had the highest AUROC. These models were based on easily obtained parameters in clinic, making them practical in application.

Despite a growing number of LT performed in China over the years, there remains increasing discrepancy between the need for transplantation and the availability of donor organs. LT is considered a life-saving treatment for ACLF patients. The current policy for organ allocation in LT is to give priority to the sickest patients mostly using MELD score in ranking. It is difficult to consider whether the ACLF patient has a favorable prognosis following LT. Use of ML will dramatically enhance the efficiency of allocation of DCD organs for LT and contribute to maximal organ utilization. The selected preoperative variables in this study consisted of six parameters, namely, Cr, INR, etiology of liver disease, DBIL, LYM and NEUT. In our study, multivariate Cox regression modeling identified that $\mathrm{Cr}$ and INR were distinct prognostic factors of poor short-term survival in association with ACLF following LT. $\mathrm{Cr}$ is the most important component of the MELD score, which can objectively reflect the severity of chronic liver diseases and prioritize liver transplant candidates. Moreover, some data indicated that higher values of $\mathrm{Cr}$ were correlated with the poor prognoses of patients with liver diseases and LT recipients [22, 25]. First, pretransplant renal function was associated with renal insufficiency and increased short-term mortality following LT following LT $[26,27]$. The occurrence of pretransplant renal function injury may result in an increase incidence of kidney failure and permanent kidney damage after LT [28]. Second, LT candidates with renal dysfunction have an increased risk of higher mortality risk from cardiovascular disease (CVD) after LT. Previous research indicated that pretransplant renal impairment was an independent indicator of post-LT CVD mortality among LT recipients [29]. The INR, a marker for coagulopathy, was one of the key components of both CLIF-C ACLFs and MELD, which emphasized the role of coagulopathy in forecasting the prognosis of patients with liver disease [30]. Our study demonstrated that the predominant etiology related to HBV (84.1\%) of ACLF had a critical role in improving survival rates, which is consistent with the previous study [31]. The liver is the 

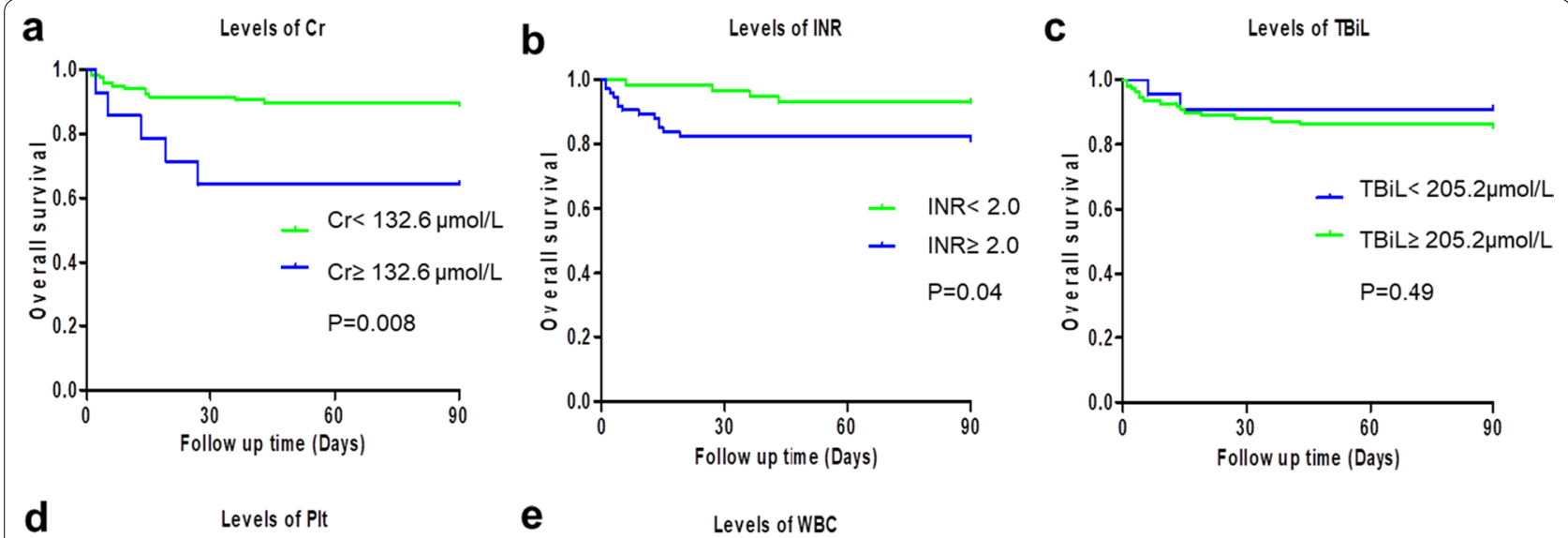

e

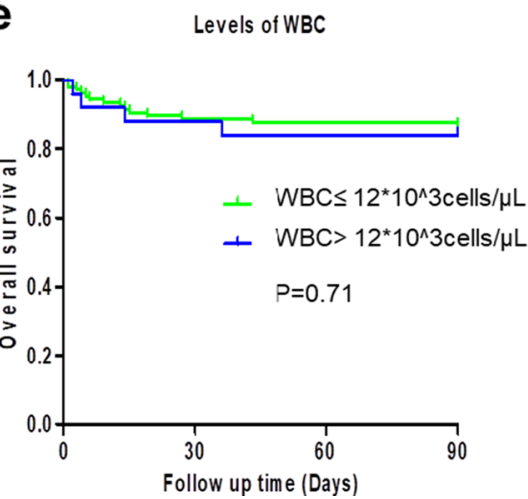

Fig. 2 The overall survival curves of patients with the Kaplan-Meier estimator, tested with a log-rank test. a The overall survival curves of patients with different levels of $\mathrm{Cr}, P=0.008$. b The overall survival curves of patients with different levels of INR, $P=0.04$. $\mathbf{c}$ The overall survival curves of patients with different levels of TBiL, $P=0.49$. $\mathbf{d}$ The overall survival curves of patients with different levels of Plt, $P=0.41$. e The overall survival curves of patients with different levels of WBC, $P=0.71$. Cr creatinine; INR International Normalized Ratio; TBiL total bilirubin; PIt platelet; WBC White Blood Cells

Table 3 Different scoring systems of the patients

\begin{tabular}{lllll}
\hline Scoring systems & ALL $(\mathbf{n}=\mathbf{1 3 2})$ & Survival $(\mathbf{n}=\mathbf{1 1 3})$ & Death $(\mathbf{n}=\mathbf{1 9})$ & $\boldsymbol{P}$ value \\
\hline MELD Score, mean \pm SD & $24.69 \pm 7.83$ & $23.94 \pm 7.44$ & $29.13 \pm 8.79$ & 0.01 \\
CLIF-OF, median (IQR) & $9.5(8-11)$ & $9(8-11)$ & $10(9-13)$ & 0.13 \\
CLIF-C ACLFs, mean \pm SD & $42.57 \pm 9.22$ & $41.89 \pm 9.19$ & $46.62 \pm 8.55$ & 0.04 \\
CLIF-SOFA, median (IQR) & $8(7-10)$ & $8(7-10)$ & $9(7-12)$ & 0.06 \\
ABIC, mean \pm SD & $8.78 \pm 1.85$ & $8.66 \pm 1.79$ & $9.53 \pm 2.06$ & 0.06 \\
\hline
\end{tabular}

Tested by Student's t-test or Mann-Whitney U test; SD Standard Deviation; IQR interquartile range; MELD Model for end-stage liver disease; CLIF-SOFA Chronic liver failure-Sequential organ failure assessment; CLIF-C OF Chronic liver failure consortium Organ Failure score; $A B I C$ age-bilirubin-international normalized ratio-creatinine

major organ of bilirubin metabolism, and hepatocytes are the only cells that can produce DBiL [32]. In ACLF patients, the higher direct bilirubin suggested moderate hepatocyte damage, consistent with the fact that DBiL was negatively correlated with prognosis in our study. Hypolymphemia and neutropenia are physiological responses to adverse stressful events, which often predict adverse outcomes of LT with ACLF. Bing-Yi Lin et al. illustrated that a high neutrophil-lymphocyte ratio indicated poor prognosis for ACLF after LT [6].
Some limitations of the study should be mentioned. Firstly, our study was a single center retrospective study. Secondly, the lack of clinical donor data may have slightly influenced the analysis results. Additionally, the small number of cases may have slightly affected the performance of the machine learning techniques. Future largescale and multicenter are required to evaluate whether better organ allocation by machine learning algorithms could promote transplant survival. 

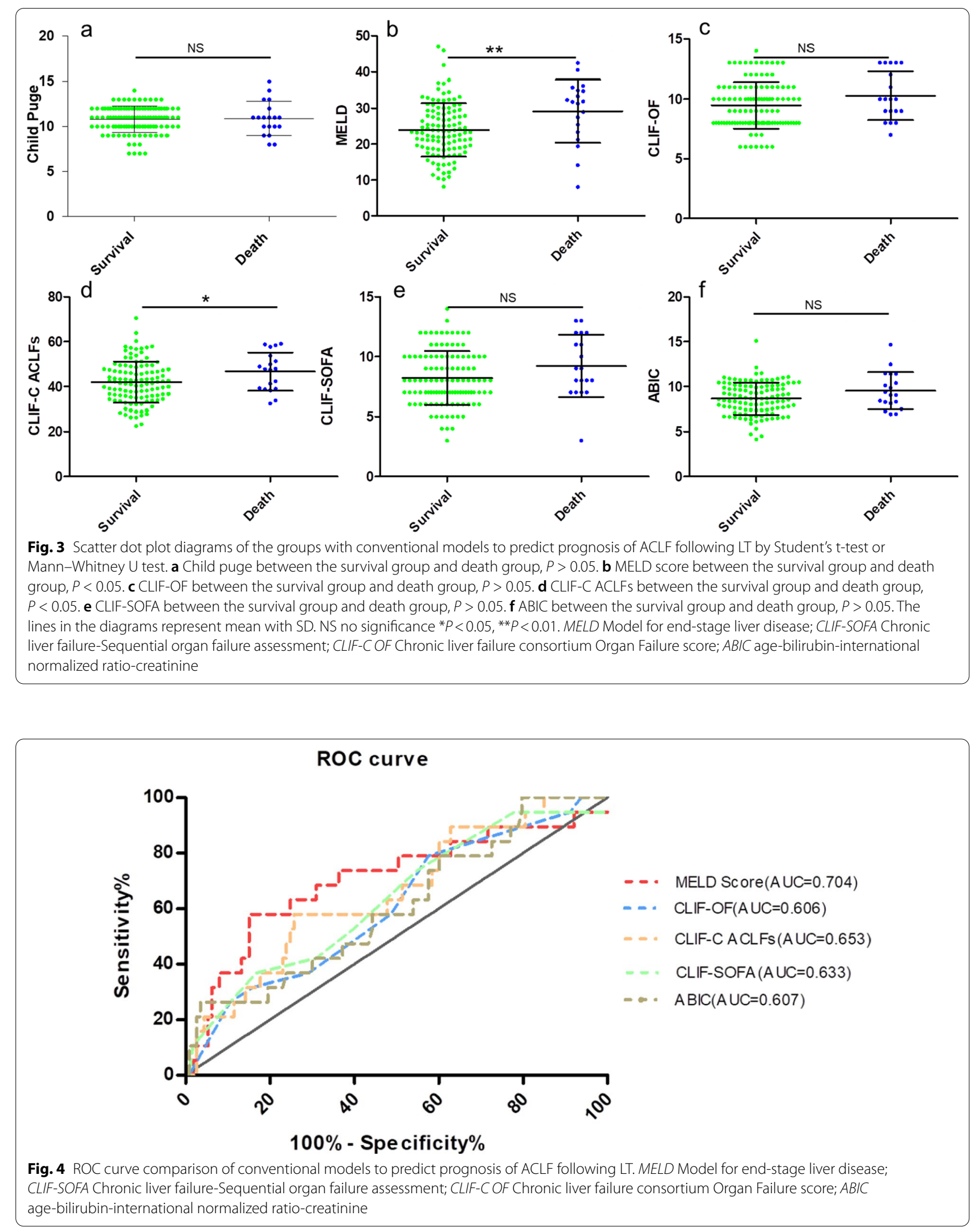


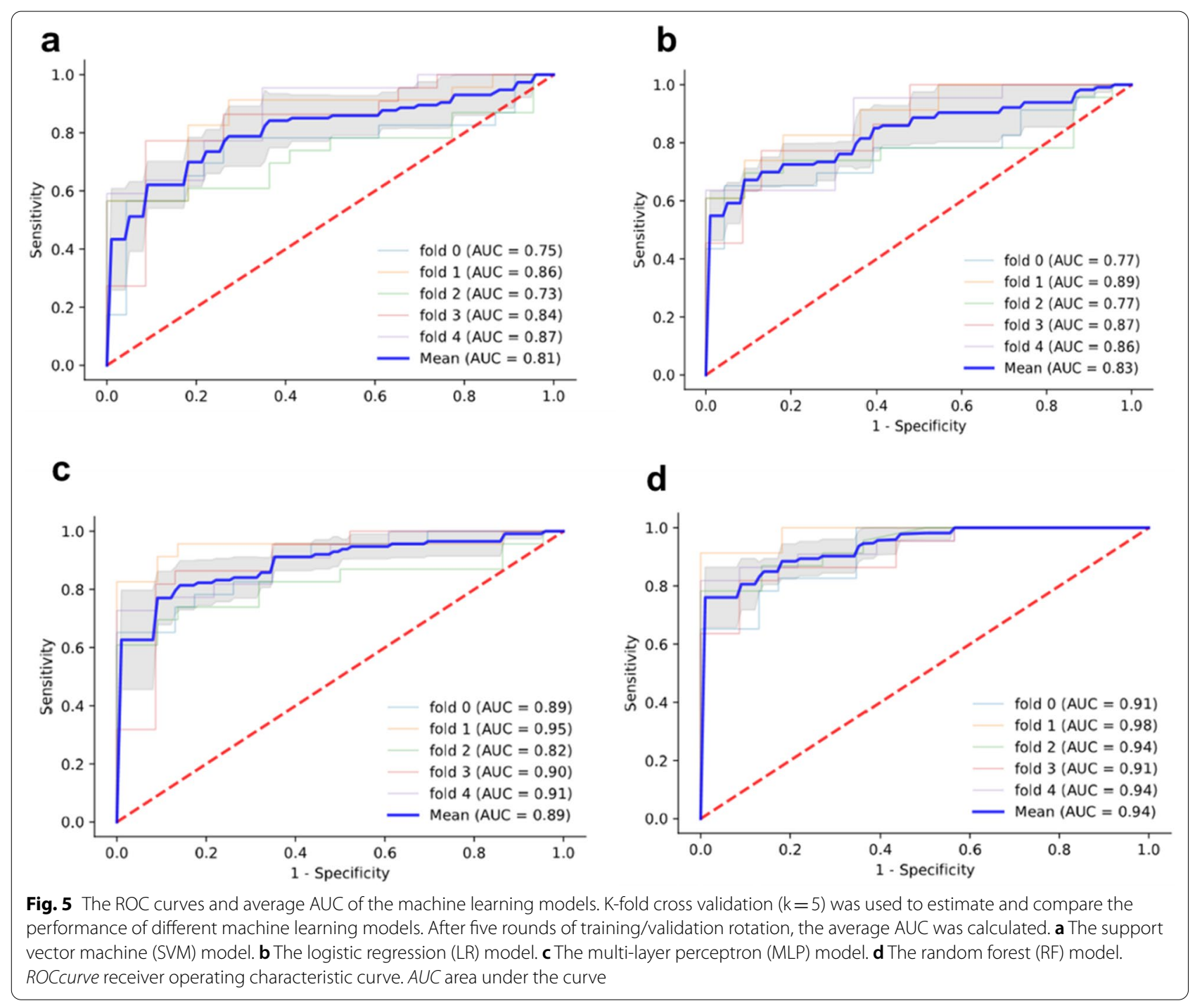

Table 4 The coefficients of the SVM and LR models

\begin{tabular}{lcc}
\hline Parameters of models & SVM & LR \\
\hline Cr & -4.24 & -3.18 \\
INR & -3.02 & -2.12 \\
HBV & 1.43 & 0.76 \\
DBiL & 1.04 & 1.10 \\
LYM & 2.15 & 1.80 \\
NEUT & -1.53 & -1.43 \\
Constant & 0.29 & 0.31 \\
\hline
\end{tabular}

SVM support vector machine; $L R$ logistic regression; $C r$ creatinine; INR International Normalized Ratio; HBV Hepatitis B Virus; DBiL Direct Bilirubin; LYM lymphocyte count; NEUT neutrophil count

\section{Conclusions}

The ML models have good performance in predicting the short-term survival of ACLF patients following LT. The RF model best predicted the prognosis. With good predictive models, better organ allocation and clinical outcome can be achieved.

\section{Supplementary Information}

The online version contains supplementary material available at https://doi. org/10.1186/s12876-022-02164-6.

Additional file 1: Supplementary Tables. 


\section{Acknowledgements}

There are no acknowledgements.

\section{Authors' contributions}

YM, BP and MY conceived and designed the study. MY collected the data. MY and $Y M$ analyzed the data and wrote the manuscript. QZ, JL, HL and KC revised the manuscript. All authors read and approved the final manuscript.

\section{Funding}

This study was supported by grants from the National Natural Science Foundation of China (81700658 and 81771722) and the Natural Science Foundation of Hunan.

\section{Availability of data and materials}

The data that support the findings of this study are available from the corresponding author upon reasonable request.

\section{Declarations}

\section{Ethics approval and consent to participate}

The study protocol complied with the standards of the Declaration of Helsinki and obtained approval from the Institutional Review Board of Third Xiangya Hospital, Central South University (No. 2020-S398). All patients provided written informed consent to participate before study enrollment.

\section{Consent for publication}

Not applicable.

\section{Competing interests}

None of the authors have competing interests to declare.

Received: 10 November 2021 Accepted: 15 February 2022 Published online: 23 February 2022

\section{References}

1. Arroyo V, Moreau R, Jalan R. Acute-on-chronic liver failure. N Engl J Med. 2020;382(22):2137-45.

2. Belli L, Duvoux C, Artzner T, Bernal W, Conti S, Cortesi P, Sacleux S, Pageaux G, Radenne S, Trebicka J et al: Liver transplantation for patients with acute-on-chronic liver failure (ACLF) in Europe: results of the ELITA/ EF-CLIF collaborative study (ECLIS). J Hepatol 2021.

3. Artru F, Louvet A, Ruiz I, Levesque E, Labreuche J, Ursic-Bedoya J, Lassailly G, Dharancy S, Boleslawski E, Lebuffe G, et al. Liver transplantation in the most severely ill cirrhotic patients: a multicenter study in acute-onchronic liver failure grade 3. J Hepatol. 2017;67(4):708-15.

4. Sundaram V, Jalan R, Wu T, Volk M, Asrani S, Klein A, Wong R. Factors associated with survival of patients with severe acute-on-chronic liver failure before and after liver transplantation. Gastroenterology. 2019;156(5):1381-1391.e1383.

5. Levesque E, Winter A, Noorah Z, Daurès JP, Landais P, Feray C, Azoulay D. Impact of acute-on-chronic liver failure on 90-day mortality following a first liver transplantation. Liver Int. 2017;37(5):684-93.

6. Lin BY, Zhou L, Geng L, Zheng ZY, Jia JJ, Zhang J, Yao J, Zheng SS. High neutrophil-lymphocyte ratio indicates poor prognosis for acute-onchronic liver failure after liver transplantation. World J Gastroenterol. 2015;21(11):3317-24.

7. Benckert C, Quante M, Thelen A, Bartels M, Laudi S, Berg T, Kaisers U, Jonas S. Impact of the MELD allocation after its implementation in liver transplantation. Scand J Gastroenterol. 2011;46(7-8):941-8.

8. Klein KB, Stafinski TD, Menon D. Predicting survival after liver transplantation based on pre-transplant MELD score: a systematic review of the literature. PLoS ONE. 2013;8(12):e80661.

9. Wagener G, Raffel B, Young AT, Minhaz M, Emond J. Predicting early allograft failure and mortality after liver transplantation: the role of the postoperative model for end-stage liver disease score. Liver Transplant. 2013:19(5):534-42.

10. Jalan R, Saliba F, Pavesi M, Amoros A, Moreau R, Ginès P, Levesque E, Durand F, Angeli P, Caraceni P, et al. Development and validation of a prognostic score to predict mortality in patients with acute-on-chronic liver failure. J Hepatol. 2014;61(5):1038-47.

11. Engelmann C, Thomsen KL, Zakeri N, Sheikh M, Agarwal B, Jalan R, Mookerjee RP. Validation of CLIF-C ACLF score to define a threshold for futility of intensive care support for patients with acute-on-chronic liver failure. Critical Care (London, England). 2018;22(1):254.

12. Chen $\mathrm{BH}$, Tseng HJ, Chen WT. Comparing eight prognostic scores in predicting mortality of patients with acute-on-chronic liver failure who were admitted to an ICU: a single-center experience. J Clin Med 2020;9(5).

13. Spann A, Yasodhara A, Kang J, Watt K, Wang B, Goldenberg A, Bhat M. Applying machine learning in liver disease and transplantation: a comprehensive review. Hepatology (Baltimore, MD). 2020;71(3):1093-105.

14. Lau L, Kankanige $Y$, Rubinstein B, Jones R, Christophi C, Muralidharan $V$, Bailey J. Machine-learning algorithms predict graft failure after liver transplantation. Transplantation. 2017;101(4):e125-32.

15. Liu CL, Soong RS, Lee WC, Jiang GW, Lin YC. Predicting short-term survival after liver transplantation using machine learning. Sci Rep. 2020;10(1):5654.

16. Lee HC, Yoon SB, Yang SM, Kim WH. Prediction of acute kidney injury after liver transplantation: machine learning approaches vs. logistic regression model. J Clin Med 2018;7(11).

17. Ayllón MD, Ciria R, Cruz-Ramírez M, Pérez-Ortiz M, Gómez I, Valente R, O'Grady J, de la Mata M, Hervás-Martínez C, Heaton ND, et al. Validation of artificial neural networks as a methodology for donor-recipient matching for liver transplantation. Liver Transplant. 2018;24(2):192-203.

18. Briceño J, Cruz-Ramírez M, Prieto M, Urbina JO, Orti R, Gómez-Bravo M-Á, Otero A, Varo E, Tomé S, Clemente G et al. Use of artificial intelligence as an innovative donor-recipient matching model for liver transplantation: results from a multicenter Spanish study. J Hepatol 2014;61(5):1020-1028.

19. Tang J, Liu R, Zhang YL, Liu MZ, Hu YF, Shao MJ, Zhu LJ, Xin HW, Feng GW, Shang WJ, et al. Application of machine-learning models to predict tacrolimus stable dose in renal transplant recipients. Sci Rep. 2017;7:42192.

20. Sarin SK, Choudhury A, Sharma MK, Maiwall R, Al Mahtab M, Rahman S, Saigal S, Saraf N, Soin AS, Devarbhavi H, et al. Acute-on-chronic liver failure: consensus recommendations of the Asian Pacific association for the study of the liver (APASL): an update. Hep Intl. 2019;13(4):353-90.

21. Peng B, Gong H, Tian H, Zhuang Q, Li J, Cheng K, Ming Y. The study of the association between immune monitoring and pneumonia in kidney transplant recipients through machine learning models. J Transl Med. 2020;18(1):370.

22. Deo SV, Al-Kindi SG, Altarabsheh SE, Hang D, Kumar S, Ginwalla MB, ElAmm CA, Sareyyupoglu B, Medalion B, Oliveira GH, et al. Model for end-stage liver disease excluding international normalized ratio (MELDXI) score predicts heart transplant outcomes: Evidence from the registry of the United Network for Organ Sharing. J Heart Lung Transplant. 2016:35(2):222-7.

23. Chen L, Zheng J, Cai J, Jie Y, Zhang Y, Li H, Lu T, He L, Xiao C, Zeng K, et al. Predictive value of age-bilirubin-international normalized ratio-creatinine score in short-term survival of acute-on-chronic hepatitis B liver failure. Cell Physiol Biochem. 2018;51(5):2484-95.

24. Asrani S, Kim W. Model for end-stage liver disease: end of the first decade. Clin Liver Dis. 2011;15(4):685-98.

25. Cabezuelo JB, Ramírez P, Ríos A, Acosta F, Torres D, Sansano T, Pons JA, Bru M, Montoya M, Bueno FS, et al. Risk factors of acute renal failure after liver transplantation. Kidney Int. 2006;69(6):1073-80.

26. Bahirwani R, Forde Ka Fau - Mu Y, Mu Y Fau - Lin F, Lin F Fau - Reese P, Reese P Fau - Goldberg D, Goldberg D Fau - Abt P, Abt P Fau - Reddy KR, Reddy Kr Fau - Levine M, Levine M. End-stage renal disease after liver transplantation in patients with pre-transplant chronic kidney disease. Clin Transplant 2014, 28(1399-0012 (Electronic)):205-210.

27. Ding Y, Wu T, Zhang W, Zhang S, Wang W. Pretransplant renal function evaluated by serum cystatin $C$ was associated with mortality after liver transplantation: a single-center experience. Ann Transl Med 2019;7(2305-5839 (Print)):243.

28. Mantovani A, Zaza G, Byrne CD, Lonardo A, Zoppini G, Bonora E, Targher G. Nonalcoholic fatty liver disease increases risk of incident chronic kidney disease: a systematic review and meta-analysis. Metabolism 2018, 79(1532-8600 (Electronic)):Metabolism, 2018, 2079:2064-2076 
29. VanWagner LB, Lapin B, Skaro Al, Lloyd-Jones DM, Rinella ME. Impact of renal impairment on cardiovascular disease mortality after liver transplantation for nonalcoholic steatohepatitis cirrhosis. Liver Int 2015;35(1478-3231 (Electronic)):2575-2583.

30. Rashidi-Alavijeh JA-O, Ceylan AS, Wedemeyer H, Kleefisch M, Willuweit K, Lange CM. Standard coagulation tests are superior to thromboelastometry in predicting outcome of patients with liver cirrhosis. PLoS One 2020;15(1932-6203 (Electronic)):e0236528.

31. Moon DB, Lee SG, Kang WH, Song GW, Jung DH, Park GC, Cho HD, Jwa EK, Kim WJ, Ha TY et al. Adult living donor liver transplantation for acute-on-chronic liver failure in high-model for end-stage liver disease score patients. Am J Transplantation 2017;17(1600-6143 (Electronic)):1833-1842.

32. Liu X, Zhang Y, Liu L, Pan Y, Hu Y, Yang P, Liao M. Protective and therapeutic effects of nanoliposomal quercetin on acute liver injury in rats. BMC Pharmacol Toxicol. 2020;21(1):11.

\section{Publisher's Note}

Springer Nature remains neutral with regard to jurisdictional claims in published maps and institutional affiliations.

- fast, convenient online submission

- thorough peer review by experienced researchers in your field

- rapid publication on acceptance

- support for research data, including large and complex data types

- gold Open Access which fosters wider collaboration and increased citations

- maximum visibility for your research: over $100 \mathrm{M}$ website views per year

At BMC, research is always in progress.

Learn more biomedcentral.com/submissions 